\title{
Verruciform xanthoma of the penis: A rare benign lesion that simulates carcinoma
}

\author{
Aldo Franco De Rose ${ }^{1}$, Mattia Tosi ${ }^{1}$, Guglielmo Mantica ${ }^{1}$, Nataniele Piol ${ }^{2}$, Carlo Toncini ${ }^{2}$, \\ Carlo Terrone $^{1}$ \\ ${ }^{1}$ Department of Urology, IRCCS San Martino, University of Genova, Italy; \\ ${ }^{2}$ Department of Pathology, IRCCS San Martino Hospital, University of Genova, Italy.
}

\begin{abstract}
Summary Verruciform xanthoma is a rare and benign condition predominantly affecting the oral cavity, but also skin and female anogenital mucosa. It can be flat, papular-warty or crateriform-cystic.

Furthermore it can simulate HPV viral lesion such as condyloma and malignant neoplasia such as verrucous squamous cell carcinoma. An accurate diagnosis is important to avoid overtreatment, considering it is a benign lesion that does not require any radical treatment. We present an extremely rare case of a 64 year-old man with a small, slighty raised, gray reddish-dotted lesion on the left portion of the ventral side of his glans.
\end{abstract}

KEY WORDS: Verruciform xanthoma; Penis.

Submitted 12 April 2016; Accepted 14 April 2016

\section{INTRODUCTION}

Verruciform xanthoma (VX) is a rare, benign lesion usually affecting oral mucosa, but also skin and sometimes anogenital mucosa, predilecting females (vulva) rather than males (scrotum, foreskin, etc.) (1). Histologically, foamy histiocytes aggregates in the subepithelial stroma or in the papillary dermis in association with verrucous epithelial acanthosis and hyperparakeratosis, are the hallmark of this lesion (2). The extra-oral (cutaneous) occurrence of VX is extremely uncommon with less than few dozen cases reported in literature, most of which occurred on anogenital areas such as vulva and scrotum. Penis involvement (foreskin or glans) is even more infrequent with very few cases described in the literature so far (3). We present a rare case of VX localized on the glans.

\section{Case report}

A 64 year-old Caucasian man, with a silent past medical history, was admitted to our institution with a small, slightly raised, grey reddish-dotted lesion on the left portion of the ventral side of his glans, previously unsuccessfully treated with antibiotic creams, antifungal therapy and antiinflammatory corticosteroid for about one month. No other similar cutaneous, genital or oral lesions were detected upon physical examination and no inguinal lymphadenopathy was present. Blood test, particularly lipid profile and serological test for sexual transmitted disease were negative both for the patient and his

partner. We decided to perform a radical excision of the lesion as outpatients under local anaesthesia.

After standard formalin fixation and paraffin embedding of the specimen, $4 \mu \mathrm{m}$ sections were cut and stained with Hematoxylin-Eosin. Histopathological examination revealed the presence of acanthosis, hyperkeratosis and parakeratosis, associated with neutrophil exocytosis and with inflammatory infiltrates of large foamy histiocytes at the apex of the dermal papillae (Figure 1).

Histiocyties were highlighted by immunohistochemical staining for CD68 + (Figure 2) and for pS100 +, thus confirming the diagnosis of VX. Negative immunostaininig for p16 ruled out a possible viral aetiology. We clinically evaluated the patient 3 months after radical excision, no recurrence of the lesion was apparent.

Figure 1.

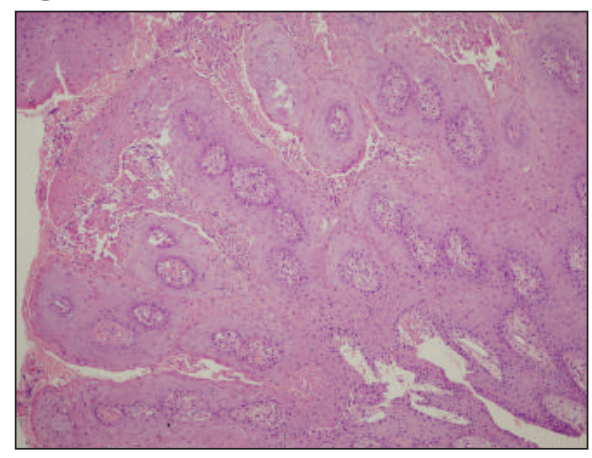

This microphotographs show an hyperplastic epithelium with papillary configuration, acanthosis and hyperparakeratosis associated with neutrophil exocytosis. No epithelial atypia is observed. Foamy histiocytes can be seen at the apex of the papillae.
Figure 2.

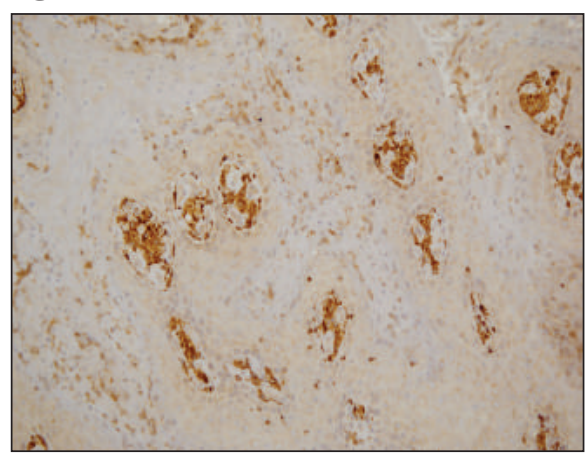

Immunostaining for CD68 PG-M1 highlights foamy histiocytes. 


\section{Discussion}

Clinically VX is a flat, raised or pebbled-warty lesion, its color being pink, greyish or yellowish depending on the degree of keratinization. It occurs mostly in adult patients in their fifth to sixth decade of life as an usually single, not sore and well-circumscribed lesion of oral mucosa, but it can also occur on anogenital areas (4).

Histologically VX is most frequently observed in its papular-warty form, but it has been described, although less frequently, in other architectural pattern such as flat and crateriform-cystic (5).

Histological features of the lesion are the same independent of architectural pattern and localization.

Epithelium has elongated rete ridges all extending in the underlying stroma to the same level and sometimes with fusion of their bases. Acanthosis, hypogranulosis and hyperparakeratosis are common findings in association with neutrophil exocytosis, especially localized between the stratum corneum and the stratum spinosum. No epithelial atypia nor koilocytosis can be found.

Subepithelial stroma shows a papillomatous pattern with thin papillae between epithelial ridges, the former with foamy histiocytes which are localized particularly at the apices of the papillae. Tlymphocytes e plasma cells, the latter usually as a band-like infiltrate under the level of elongated rete ridges, are usually present (6).

VX was described for the first time in 1971 by Shafer in its oral localization and in 1979 by Santa Cruz in extraoral site; particularly VX of penis it was firstly described by Kraemer et colleagues in 1981 (7). Its etiopathogenesis is still nowadays not entirely known; viral causes having been investigated, and in particular a correlation with HPV infection, because of similar clinical and histological similarities between VX and HPV-related lesions such as condylomata (8-9). A second pathogenetic hypothesis concerns the correlation with lipid disorders, but many patients do not show alterations in their clinical and laboratory exams to support this possibility (10). Today the most quoted hypothesis is that VX can have a traumatic pathogenesis, namely keratinocytes disruption due to traumatic injury primes the establishment of an inflammatory status which typically attracts $\mathrm{T}$ lymphocytes and neutrophils and later macrophages in order to degrade damaged cellular debris thus becoming foamy histiocytes (10). Differential diagnosis includes above all viral (HPV) and malignant lesions (11), VX sharing morphological similarities both with condylomata and with verrucous squamous cell carcinoma. A correct diagnosis is fundamental, especially excluding the possibility of cancer, because VX is a benign lesion that does not require any radical treatment and so any over-treatment should be avoided (12).

\section{Conclusion}

Recognition of genital VX is important to avoid unnecessary investigations or surgical procedures and to reduce patient's anxiety. To diagnose VX is essential to perform a biopsy for histoplatologic examination. Microscopy features allow for a distinction between VX and squamous cell carcinoma, which is included in its differential diagnosis. The treatment of VX typically involves a simple surgical excision.

\section{REFERENCES}

1. Mohsin SK, Lee MW, Amin MB, et al. Cutaneous verruciform xanthoma: a report of five cases investigating. Am J Surg Pathol. 1998; 22:479-87.

2. Cobb CM, Holt R, Denys FR. Ultrastructural features of the verruciform xanthoma. J Oral Pathol. 1976; 5:42-52.

3. Sinnya S, Wheller L, Carroll M, et al. Verruciform xanthoma of the penis: a rare Australian case. Australas J Dermatol. 2015; 56:99-101.

4. Xia TL, Li GZ, Na YQ, et al. Verruciform xanthoma of the penis: report of a case. Chin Med J. 2004; 117:150-2.

5. Philipsen HP, Reichart PA, Takata T, et al. Verruciform xanthoma: Biological profile of 282 oral lesions based on literature and survey with nine new casesfrom Japan. Oral Oncol. 2003; 39:325-36.

6. Woo S-B. Diseases of the oral mucosa. In: McKee PH, Claonje E, Granter SR. Pathology of the skin with clinical correlations. 3rd ed. Volume 1. Philadelphia: Elsevier Mosby, 2005; pp 412-413.

7. Kraemer BB, Schmidt WA, Foucar E, et al. Verruciform xanthoma of the penis. Arch Dermatol. 1981; 117:516-518.

8. Khaskhely NM, Uezato H, Kamiyama T, et al. Association of human papilloma virus type 6 with verruciform xanthoma. Am. J. Dermatopathol. 2000; 22:447-52.

9. Rohwedder A, Murphy M, Carlson FA. Multiple human papillomavirus DNA identified in verruciform xanthoma by nested polymerase chain reaction with degenerate consensus primers. J. Cutan. Pathol. 2003; 30:344-6.

10. Zegarelli DJ, Zegarelli-Schmidt EC, Zegarelli EV. Verruciform xanthoma: A clinical, light microscopic and electron microscopic study of two cases. Oral Surg. 1974; 38:725-34.

11. Takiwaki H, Yokota M, Ashan K, et al. Squamous cell carcinoma associated with verruciform xanthoma of the penis. Am. J. Dermatopathol. 1996; 18:551-4.

12. Astori G, Lavergne D, Benton C, et al. Human papillomaviruses are commonly found in normal skin of immunocompetent hosts. Actas Dermosifiliogr 2008; 99:75-6.

\section{Correspondence}

Aldo Franco De Rose, MD

aldofdr@libero.it

Mattia Tosi, MD

matti.grifone@hotmail.it

Guglielmo Mantica, MD

Carlo Terrone, MD

Department of Urology, IRCCS San Martino, University of Genova,

Genova, Italy

Nataniele Piol, MD

Carlo Toncini, MD

Department of Pathology, IRCCS San Martino Hospital,

University of Genova, Genova, Italy 